\title{
You can't always get what you want
}

\author{
Harold G. Roberts, MD, Lawrence M. Wei, MD, and Vinay Badhwar, MD \\ From the Department of Cardiovascular and Thoracic Surgery, West Virginia University, Morgantown, WVa. \\ Disclosures: The authors have nothing to disclose with regard to commercial support. \\ Received for publication Sept 3, 2017; accepted for publication Sept 8, 2017; available ahead of print Oct 15, \\ 2017. \\ Address for reprints: Vinay Badhwar, MD, Department of Cardiovascular and Thoracic Surgery, West Virginia \\ University, 1 Medical Center Drive, Morgantown, WV 26506-8059 (E-mail: vinay.badhwar@wvumedicine. \\ org). \\ J Thorac Cardiovasc Surg 2018;155:103-4 \\ $0022-5223 / \$ 36.00$ \\ Copyright (C) 2017 by The American Association for Thoracic Surgery \\ https://doi.org/10.1016/j.jtcvs.2017.09.034
}

In the current issue of the Journal, Schnittman and colleagues ${ }^{1}$ provide an elegant retrospective analysis of isolated mitral valve replacement (MVR) operations in patients aged 18 to 50 years using New York and California administrative claims data. They found an alarming overall trend in the increase in bioprostheses (BPs) from 10\% to $34 \%$ between 1997 and 2014. After excluding endocarditis and congenital abnormalities, they laudably examined 373 comprehensively propensity-matched pairs of isolated MVR receiving mechanical prostheses (MPs) versus BP from 1997 to 2006 to assess 15-year survival and readmissions for stroke, major bleeding, and valve reoperation. The key findings were (1) a 20-fold increase in MVR reoperation of BP versus MP; (2) a significant survival difference in favor of MP (hazard ratio, 1.67); and (3) no difference in the longitudinal rate of stroke or bleeding.

The 30-year precedent of mitral MP durability over BP established by the Veterans Affairs randomized trial also documented bleeding rates associated with anticoagulation. ${ }^{2}$ Nevertheless, MP remains the standard of care for MVR in younger patients. For older patients requiring aortic valve replacement, improved contemporary BP durability has secured it as the most common therapy. Recent institutional trends of possible midterm suitability of BP use in younger patients requiring aortic valve replacement have ignited a trend of seductive convenience for both patients and physicians to request $\mathrm{BP}$ as a potentially acceptable anticoagulation avoidance strategy. In the current study, ${ }^{1}$ the authors identify the commencement of an early trend of BP use in young patients receiving MVR.

Contemporary isolated mitral operations represent 5.5\% of all adult cardiac operations performed in the United States. $^{3}$ They have a risk-adjusted mortality of $2.9 \%$ (1.0\% for mitral repair) and a 30-day stroke rate of $1.5 \%$, $14.5 \%$ are performed via nonsternotomy or robotic techniques, and $75 \%$ of degenerative valves are now repaired. ${ }^{4}$ Whenever anatomically feasible, it is accepted that repair is superior to replacement to secure optimal long-term outcomes. So, when interpreting a relatively contemporary analysis describing increasing rates of $\mathrm{BP} \mathrm{MVR}^{1}$, one

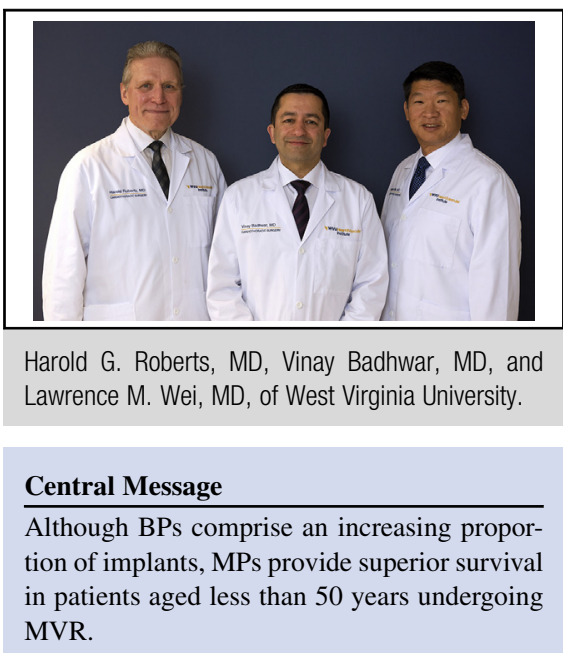

See Article page 96.

would expect this to describe a high-risk population with blunted life expectancy or incapacity for anticoagulation. Although bias to the latter of course may have merit, the current study ${ }^{1}$ focuses on young patients in whom a blunted life expectancy explanation is less likely, and the propensity-matched findings excluded confounders of active endocarditis or congenital disease. Although the authors $^{1}$ dutifully note reasons of a BP trend to include pregnancy choice and other possible risks, this somewhat languid explanation appears insufficient to punctuate the importance of these data. Noting an MP survival curve separation at approximately 8 years without bleeding or stroke differences to BP appears to negate the merit of anticoagulation avoidance in young patients. This finding corroborates other propensity-matched observations noting that MP may confer survival benefits to young patients as early as 7.5 years postoperatively, before expected structural valve degeneration. ${ }^{5}$ Recent echocardiographic evidence suggests early BP MVR thrombosis in young patients is more common than previously reported, occurring before structural degeneration and with characteristic gradient increases after 2 years. ${ }^{6}$ This may be further explained by the finding that patients with BPs have highly abnormal patterns of postoperative intraventricular flow vortices compared with repair or patients with MPs.

For young patients of otherwise appropriate reliability and medical stability, this study ${ }^{1}$ serves as a clarion call to arrest an incipient trend of BP implantation that is unsupported by evidence. When confronted with a young 
patient with mitral disease without contraindications to anticoagulation deemed not repairable, despite the desire to avoid warfarin, we must use the evidence to counsel patients of their need of a mechanical valve by helping them to understand that "you can't always get what you want, but if you try sometimes you just might find you get what you need!" 8

\section{References}

1. Schnittman SR, Itagki S, Toyada N, Adams DH, Egoravo NN, Chikwe J. Survival and long-term outcomes after mitral valve replacement 1 in patients aged 18 to 50 years. J Thorac Cardiovasc Surg. 2018;155:96-102.e11.

2. Hammermeister K, Sethi GK, Henderson WG, Grover FL, Oprian C, Rahimtoola SH. Outcomes 15 years after valve replacement with a mechanical versus a bioprosthetic valve: final report of the Veterans Affairs randomized trial. J Am Coll Cardiol. 2000;36:1152-8.
3. D'Agostino RS, Jacobs JP, Badhwar V, Paone G, Rankin JS, Han JM, et al. The Society of Thoracic Surgeons adult cardiac surgery database: 2017 update on outcomes and quality. Ann Thorac Surg. 2017;103:18-24.

4. Badhwar V, Rankin JS, He X, Jacobs JP, Gammie JS, Furnary AP, et al. The Society of Thoracic Surgeons mitral repair/replacement composite score: a report of The Society of Thoracic Surgeons Quality Measurement Task Force. Ann Thorac Surg. 2016;101:2265-71.

5. Badhwar V, Ofenloch JC, Rovin JD, van Gelder HM, Jacobs JP. Noninferiority of closely monitored mechanical valves to bioprostheses overshadowed by early mortality benefit in younger patients. Ann Thorac Surg. 2012;93:748-53.

6. Egbe AC, Pislaru SV, Pellikka PA, Poterucha JT, Schaff HV, Maleszewski JJ, et al. Bioprosthetic valve thrombosis versus structural failure: clinical and echocardiographic predictors. J Am Coll Cardiol. 2015;66:2285-94.

7. Nakashima K, Itatani K, Kitamura T, Oka N, Horai T, Miyazaki S, et al. Energy dynamics of the intraventricular vortex after mitral valve surgery. Heart Vessels. April 7, 2017 [Epub ahead of print].

8. Richards K, Jagger M, Johnson R. You Can't Always Get What You Want. Let It Bleed. London Records, December 5, 1969. 